\title{
An Agent-Based Computer Simulation on Banana Bunchy Top Disease
}

\author{
Ador R. Picaza $^{1}$, Douglas M. Doloriel ${ }^{2}$ \\ ${ }^{1}$ University of Southern Philippines \\ ${ }^{2}$ Surigao del Sur State University-Tagbina Campus
}

\begin{abstract}
Banana Bunchy Top Disease (BBTD) is an aphid-transmitted virus disease of banana plants. Banana Bunchy Top Virus (BBTV) is the causal microorganism of this disease. Infected plants rarely produce a fruit bunch after infection and do not fruit in subsequent years. This paper studied the factors that defined the rate of BBTD spread in this $2 \times 3$ factorial simulation experiment. Results indicate that within four months at the onset of infection, the presence of vector aphids with mild (25\%) and severe (75\%) infectiousness of BBTD can infect slightly more than half and almost all banana plants in a hectare plantation, respectively.
\end{abstract}

Keywords- banana plants, Banana Bunchy Top Disease (BBTD), Banana Bunchy Top Virus (BBTV), mortality, infectiousness, symptoms.

\section{INTRODUCTION}

The word "banana" is a broad term embracing some species or hybrids in the genus Musa of the family Musaceae. The banana plant is a large herb having a pseudostem with a cylinder of leaf-petiole sheaths reaching a height of 20 to 25 feet originating from a fleshy rhizome or corm. They are primarily produced for their fruit (technically a 'berry') (Morton, 1987).

Bananas are among the world's major food crops. It is considered as the poor man's fruit crop in tropical and sub-tropical countries. They are essential plants in the subsistence diet of the poor millions. They are also important export commodities of some developing countries in Africa, Latin America, Asia and the Pacific Regions (FAO, 2004). However, its production is hampered by pests and diseases such as the Banana Bunchy Top Disease (BBTD).

BBTD is an aphid-transmitted virus disease of banana plants. Banana Bunchy Top Virus (BBTV) is the causal microorganism of this disease. Leaves formed after infection are narrow, short with upturned margins and become stiff and brittle. The leafstalks are short and unbending and remain erect, giving a 'rosetted' appearance. The leaves of suckers and three (3) youngest leaves of mother plant show yellowing and waviness or margins. The youngest leaves exhibit very narrowly, dark-green, interrupted (“dot-and-dash") lines on the underside (Morton, 1987).

Diseased plants rarely produce a fruit bunch and do not fruit in succeeding years. Plants infected late in the growing period may fruit once, but the bunch stalk and the fruit will be small and distorted. In plants infected very late, the only symptoms present may be a few dark green streaks on tips of flower bracts (Thomas et al., 1994).

Studies above have shown that the spread of BBTD in a banana plantation can be influenced by several factors. These factors include sanitation, the infectiousness of BBTV and population of aphid vectors.

The model of the study was generated from the software called NetLogo (version 5.2.1) (Wilensky, 1998), an agent-based programming language and integrated modeling environment (Kornhauser et al., 2007). The study aims to define BBTD spread on banana plants in a hectare plantation.

\section{MODEL DEFINITION}

The BBTD Model relies on the standard planting density of banana plants in a banana plantation, standard labor requirements, management practices, etc. It identifies the potent infecting nature of BBTD where it can infect $100 \%$ of banana plants.

The model relies on the following basic assumptions:

$>$ that banana plants are planted in a standard planting density (2,000 hills/hectare)

$>$ that aphids are vectors of the disease and are already present in the plantation

$>$ that aphids can transmit BBTV in 2 hours

$>$ that BBTD can infect $100 \%$ of the plants

$>$ that symptoms could appear about a month after infection

$>$ that mortalities could be observed about 4-6 months after onset of symptoms

The model simulates the scenario starting with the aphid vector that gets inside the banana plantation. The aphid with the BBTV infects a healthy banana plant. The banana plant acquires the virus and eventually shows 
the symptoms. Other aphids feed on the diseased plant and acquire the virus. They then infect other healthy plants as they forage for nutrients. This scenario is shown below:

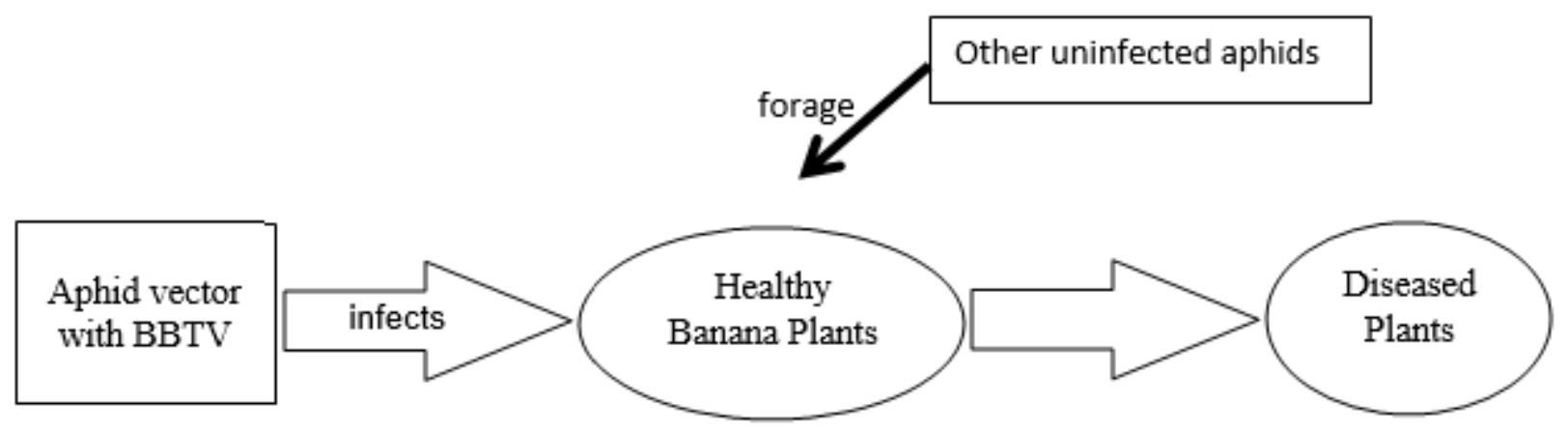

Fig.1: Schematic Diagram of the Scenario

\section{PARAMETERS}

This study used existing Virus Model of Uri Wilensky, found in the net logo models library with the following changes in parameter definition, shown in Table 1.

There are four (4) parameters that are included in the Virus Model. These parameters are Number People, Infectiousness, Chance-Recover and Duration.

One (1) parameter was added to the BBTD Model. The parameters in the BBTD Model are Initial Number of Hills, Density of Vector Aphids, Infectiousness, Chance-Recover, and Duration.

The Density of Vector Aphids was included because it is a means wherein BBTD infection could be disseminated in a plantation.

Table.1: Analysis on the Parallelism of Parameters Used in Different Model

\begin{tabular}{|l|l|}
\hline $\begin{array}{c}\text { Parameters in Virus } \\
\text { Model }\end{array}$ & \multicolumn{1}{c|}{ Parameters in BBTD Model } \\
\hline Number People & Initial Number of Hills \\
\hline & Density of Vector Aphids \\
\hline Infectiousness (\%) & Infectiousness (\%) \\
\hline Chance-Recover (\%) & Chance-Recover (\%) \\
\hline Duration (weeks) & Duration (weeks) \\
\hline
\end{tabular}

\section{RESEARCH DESIGN AND METHODS}

In this study, the variable of interest is the percentage of banana plants infected with BBTD virus at any given time. This shows the virulence of BBTD and its infection rate in a hectare plantation.

Since the study suggests that the rate of BBTD spread is influenced by the density of vector aphids and its infectiousness, we set up a computer simulation experiment. In this simulation, we controlled the indicators that are necessary for the movement of BBTD such as (A) density of vector aphids and (B) infectiousness and observed the percentage of BBTD infection under each combination of indicators. ChanceRecover and Duration indicators were fixed at $25 \%$ and 52 weeks, respectively.

Indicator (A) density of vector aphids is set at three (3) levels: low (3\%), medium (10\%) and high $(30 \%)$. Indicator (B) infectiousness is set at two (2) levels: mild (25\%) and severe (75\%). An initial number of banana plants was fixed at 2,000 hills.

This experiment is a $2 \times 3$ factorial. This factorial experimental design yields six (6) treatment combinations. Thirty (30) observations were generated to allow approximation of interaction effects of various levels of indicators. This brings the total sample size to 180 random observations.

For each combination of the two factors, the simulation generates an initial number of infected banana plants. This number is obtained from a uniform probability distribution on the interval [ 3, 10 and 30 for (A) and 25 and 75 for (B) ].

The data obtained were subjected to a two-way analysis of variance (ANOVA) with interaction effects. Two (2) main effects were measured. A and B interaction effects were observed which was A X B. Each of these effects specified the combination of indicators that led to faster BBTD infection rates.

\section{RESULTS AND DISCUSSION}

Table 2 shows the data collected from the simulation of BBTD infection rate in banana plants using the two indicators.The Density of Vector Aphids (A) with three (3) levels and Infectiousness (B) with two (2) levels. 


\begin{tabular}{|c|c|c|c|c|c|c|c|c|c|c|c|c|c|c|c|c|}
\hline & \multicolumn{15}{|c|}{ Density of Vector Aphids (A) } \\
\hline & & \multicolumn{5}{|c|}{ Low $(3 \%)$} & \multicolumn{5}{|c|}{ Medium (10\%) } & \multicolumn{5}{|c|}{ High $(30 \%)$} \\
\hline \multirow{12}{*}{ 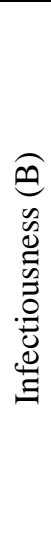 } & \multirow{6}{*}{ 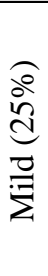 } & 41.9 & 39.9 & 56.8 & 65.4 & 57 & 62.4 & 64.4 & 60 & 51.8 & 57.1 & 52.8 & 52.8 & 55.3 & 59.6 & 43.2 \\
\hline & & 67.9 & 60.9 & 48.1 & 48.9 & 56.3 & 46.4 & 60.8 & 52.6 & 49.1 & 62.1 & 50.1 & 65 & 58.5 & 59.2 & 49.3 \\
\hline & & 54 & 53.5 & 66.2 & 45.1 & 49.9 & 65.6 & 49.8 & 45.3 & 66.9 & 41.1 & 53.8 & 53.9 & 51.4 & 51.2 & 57.2 \\
\hline & & 52.6 & 53.5 & 48.2 & 49.5 & 51.9 & 60.9 & 50.7 & 49.5 & 60 & 49.3 & 54.2 & 60.7 & 65.6 & 52.8 & 65.7 \\
\hline & & 47.9 & 67.1 & 71.4 & 60.5 & 63.8 & 41.3 & 45.9 & 62 & 61.9 & 54.3 & 61.9 & 48.3 & 62.4 & 47.3 & 53.7 \\
\hline & & 57.3 & 54.4 & 57.1 & 49.1 & 60 & 58.7 & 50.2 & 60.9 & 55.7 & 49.9 & 57.8 & 58.3 & 63.9 & 59.9 & 74 \\
\hline & \multirow{6}{*}{$\begin{array}{l}\frac{8}{2} \\
\stackrel{0}{\sigma}\end{array}$} & 100 & 100 & 100 & 100 & 99.9 & 100 & 100 & 99.6 & 99.8 & 99.7 & 99.8 & 99.9 & 99.9 & 100 & 99.8 \\
\hline & & 99. & 100 & 99.8 & 99.9 & 99.9 & 99.9 & 99.8 & 100 & 100 & 99.9 & 99.8 & 99.9 & 99.9 & 99.7 & 100 \\
\hline & & 99.9 & 99.9 & 99.6 & 99.9 & 98.7 & 99.3 & 99.9 & 99.9 & 99.9 & 99.8 & 100 & 100 & 99.8 & 99.8 & 99.9 \\
\hline & & 100 & 100 & 99.9 & 99.9 & 98.7 & 99.8 & 99.6 & 99.9 & 100 & 99.9 & 99.7 & 99.9 & 100 & 100 & 100 \\
\hline & & 100 & 99.9 & 99.8 & 99.9 & 99.8 & 99.6 & 99.8 & 100 & 99.9 & 99.9 & 99.9 & 99.9 & 100 & 99.5 & 100 \\
\hline & & 99.8 & 99.8 & 99.9 & 99.9 & 99.9 & 99.8 & 100 & 99.6 & 99.2 & 99.3 & 99.9 & 100 & 100 & 100 & 99.9 \\
\hline
\end{tabular}

Table 3 shows the two-way table for the effects of Factor A (density of vector aphids) and Factor B (infectiousness).

Table.3: Effect of Density of Vector Aphids and Infectiousness(\%) on Rate of BBTD Spread

\begin{tabular}{|c|c|c|c|c|}
\hline & \multicolumn{3}{|c|}{ Density of Vector Aphids (A) } \\
\hline & & Low $(3 \%)$ & Medium (10\%) & High $(30 \%)$ \\
\hline$\stackrel{\infty}{\infty}$ & 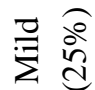 & 55.7 & 54.9 & 56.7 \\
\hline 总 & 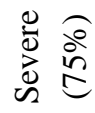 & 99.9 & 99.8 & 99.9 \\
\hline
\end{tabular}

Results show that high density of vector aphids (30\%) coupled with mild infectiousness $(25 \%)$ resulted in a $56.7 \%$ spread of BBTD in an hectare of banana plantation after 4 months at the onset of infection followed by low density $(3 \%)$ of vector aphids with mild infectiousness (25\%) having $55.7 \%$ and medium $(10 \%)$ density coupled with mild infectiousness with $54.9 \%$ BBTD spread. Moreover, these vector aphids retain the virus for weeks and could cover long distances through wind and rain dispersion (Balcaen, 2016). However, the different levels of density of vector aphids have no significant effect on the rate of infection.

Severe infectiousness $(75 \%)$ coupled with either low $(3 \%)$ or high $(30 \%)$ density of vector aphids resulted in $99.9 \%$ BBTD spread. Severe infectiousness coupled with medium density $(10 \%)$ of vector aphids resulted to $99.8 \%$ disease spread and is not significantly lower than having low (3\%) and high (30\%) density of vector aphids.

Table.4. Two-way ANOVA for Banana Bunchy Top Disease

\begin{tabular}{lccccc}
\hline \multicolumn{1}{c}{ Sources } & $d \mathrm{f}$ & $\mathrm{SS}$ & $\mathrm{MS}$ & $F$ & $p-$ value \\
\hline $\begin{array}{l}\text { Main Effects } \\
\quad \text { Density of Vector Aphids (A) }\end{array}$ & 2 & 26.4 & 13.2 & 0.48 & 0.621 \\
$\quad$ Infectiousness (B) & 1 & 87516.5 & 87516.5 & 3167.56 & 0.000 \\
Interaction Effects & & & & & \\
$\quad$ Treatment A x B & 2 & 21.0 & 10.5 & 0.38 & 0.685 \\
Error & 174 & 4807.4 & 27.6 & & \\
\hline Total & 179 & 92371.3 & &
\end{tabular}


Table 4 shows the two-way ANOVA for BBTD spread. Different levels of density of vector aphids (A) have no significant effect on the rate of BBTD spread as shown by the P-value (0.621) that is greater than the F value of 0.48 .

With the presence of vector aphids even with only mild infectiousness (25\%) of BBTD, 55 to $56 \%$ infection rate in a banana plantation after four months will have a significant economic impact on its productivity.

The levels of infectiousness have a significant impact on the rate of BBTD spread, i.e. P-value of 0.000 is less than its $F$ value of 3167.56 . Severe infectiousness (75\%) showed significantly higher BBTD spread (99.9\%) compared to mild infectiousness (55.8\%). Mild infectiousness is expressed in some banana cultivars and other Musa species characterized by the absence of darkgreen leaf and petiole streaks (Magee, 1953).Interaction (A X B) of the density of vector aphids (A) and infectiousness (B) have no significant effect on the rate of BBTD spread in a banana plantation.

\section{CONCLUSION AND RECOMMENDATIONS}

Within four months at the onset of infection, the presence of vector aphids with mild $(25 \%)$ and severe (75\%) infectiousness of BBTD can infect slightly more than half and almost all banana plants in a hectare plantation, respectively.

The rate of BBTD spread can be averted with quick management response to minimize disease spread. Integrated pest management (IPM) can be used to rid of vector aphids. The combination of eliminating diseased plants (physical) and usage of virus-tested planting materials (cultural method), aphicides (chemical) and use of parasitoids (biological) are some of the methods that can be applied to control aphids that vector BBTD.

\section{REFERENCES}

[1] Balcaen, A. (2016). The impact of PeVYV on its aphidvector's population growth anddispersal. Master's dissertation. Universiteit Gent. https://lib.ugent.be/fulltxt/RUG01/002/272/553/RU G01-002272553_2016_0001_AC.pdf

[2] FAO. (2004).Cellular Biology and Biotechnology Including Mutation Techniques for Creation of New Useful Banana Genotypes. http://www.fao.org/docrep/007/ae216e/ae216e02.ht $\mathrm{m}$

[3] Kornhauser, D, Wilensky, U., and Rand, W. (2009). Design Guidelines for Agent-Based Model Visualization. Journal of Artificial Societies and Social Simulation. JASSS, 12 (2), 1.

[4] Magee, C.J.P.(1953). Some aspects of the bunchy top disease of banana and other Musa spp. Journal and Proceedings of the Royal Society of New South Wales, 87: 3-18.

[5] Morton, J. (1987). Banana: Fruits of Warm Climates. Miami, Florida. pp. 29-46.

[6] Thomas, J.E., Iskra-Carvana, M.L., andJones, D.R. (1994). Banana Bunchy Top Disease. Musa Fact Sheet No. 4. Montpellier, France: INIBAP.

[7] Wilensky, U. (1998). Netlogo Virus Model. Center for Connected Learning and Computer-Based Modelling. Northwestern University, Evanstan, Illinois.

http://ccl.northwestern.edu/netlogo/models/Virus. 